\title{
Role of Nicotinamide N-Methyltransferase in Dorsal Striatum in Cocaine Place Preference
}

\author{
Li Luo',2,4, Fei-Fei Shang', ${ }^{3,4}$ Hailei Long', Linhong Jiang', Ruiming Zhu', Qian Zhao', Hui Gu', Jueying Kong', \\ Wei Xu', Yinglan Zhao' and Xiaobo Cen ${ }^{*}$, \\ 'National Chengdu Center for Safety Evaluation of Drugs, State Key Laboratory of Biotherapy and Cancer Center, West China Hospital, Sichuan \\ University, and Collaborative Innovation Center for Biotherapy, Chengdu, China; ${ }^{2}$ Chongqing Foreign Language School, Chongqing, China; ${ }^{3}$ Institute \\ of Life Sciences, Chongqing Medical University, Chongqing, China
}

\begin{abstract}
Nicotinamide N-methyltransferase (NNMT) transfers the methyl from S-adenosyl-L-methionine (SAM) to nicotinamide (NA) to produce S-adenosyl-L-homocysteine (SAH) and I-methylnicotinamide (MeN). NNMT has been implicated in a variety of diseases; however, the role of NNMT in drug addiction is largely unknown. Here, we found that the expression of Nnmt was significantly upregulated in the dorsal striatum (DS) of cocaine-conditioned mice. Cocaine significantly decreased SAM/SAH ratio levels in the DS, which was accompanied with the decreased activities of Racl and RhoA. Lentivirus-mediated knockdown of Nnmt in the dorsomedial striatum (DMS) attenuated cocaine conditioned place preference (CPP) reward, but increased striatal SAM/SAH ratio levels as well as Racl and RhoA activities. In addition, pharmacological inhibition of NNMT through intra-DMS infusion of MeN attenuated cocaine CPP and the activities of Racl and RhoA, but increased SAM/SAH ratio. These results suggest that NNMT-dependent transmethylation is involved in the activation of Rac I and RhoA, which utilize SAM as a methyl donor cofactor. Co-immunoprecipitation assay using a RhoGDl $\alpha$ antibody indirectly captured Racl or RhoA that were bound to RhoGDl $\alpha$. The results showed that cocaine increased the association of RhoGDl $\alpha$ with Racl or RhoA, whereas such effect was inhibited by Nnmt knockdown. Collectively, our findings show that NNMT regulates cocaine CPP through SAM-mediated modification of Racl and RhoA.
\end{abstract}

Neuropsychopharmacology (2017) 42, 2333-2343; doi:I0.1038/npp.2017.147; published online 9 August 2017

\section{INTRODUCTION}

Nicotinamide $N$-methyltransferase (NNMT), a cytoplasmic methyltransferase, transfers the methyl from $S$-adenosyl-Lmethionine (SAM) to nicotinamide (NA) to produce $S$-adenosyl-L-homocysteine (SAH) and 1-methylnicotinamide (MeN) (Aksoy et al, 1994; Riederer et al, 2009). SAM is the sole methyl donor in many important methylation reactions in nervous system, which is involved in the modification of both proteins and DNA. NNMT affects the methylation potential in multiple diseases by consuming methyl units from SAM to create a stable metabolic product MeN (Kraus et al, 2014; Sperber et al, 2015; Ulanovskaya et al, 2013). Studies have revealed the link between NNMT dysregulation and brain disorders. For instance, single nucleotide mutation of Nnmt was reported in schizophrenia patients (Wang et al, 2014), and NNMT is significantly upregulated in Parkinson's disease (Parsons et al, 2002).

\footnotetext{
*Correspondence: Dr X Cen, National Chengdu Center for Safety Evaluation of Drugs, State Key Laboratory of Biotherapy and Cancer Center, West China Hospital of Sichuan University, Gaopeng Street, Chengdu 61004I, China, Tel: +86 28 85178767, Fax: +86 28 85 |73043, E-mail: xbcen@scu.edu.cn

${ }^{4}$ These authors contributed equally to this work.

Received 7 November 20 I6; revised 9 July 20 17; accepted I 3 July 20 I7; accepted article preview online 20 July 2017
}

Nnmt expression in SH-SY5Y cells increases neurite branching, synaptophysin expression, and dopamine accumulation, which is mediated by MeN (Thomas et al, 2013). These results suggest that dysregulation of NNMT and its metabolic product are involved in neuropsychiatric disorders. However, the role of NNMT in drug-induced neuroplasticity is largely unknown.

Cytoskeleton regulation of dendritic signaling and morphology has been thought as an important mediator of plasticity associated with neuropsychiatric disorders (Penzes et al, 2011). It has been known that cocaine increases the densities of dendritic spine and promotes dendritic arborization in neurons (Christian et al, 2016; Nyberg, 2014; Robinson and Kolb, 1999). Racl and RhoA, two major members of Rho GTPase family, regulate neurite branching through controlling the movement of actin, which is critical for neural plasticity (Bradke and Dotti, 2000). Extracellular inhibitory signal molecules activate Racl and RhoA to bind to GTP, activate ROCK, and eventually inhibit neurite growth by influencing actin myosin system. On the contrary, after GTP is hydrolyzed to GDP, Racl and RhoA become inactivated (Jaffe and Hall, 2005; Tiedje et al, 2008). Rho GDP dissociation inhibitor $\alpha$ (RhoGDI $\alpha$ ) blocks the dissociation of GDP from Racl and RhoA, making them inactive, which enhances the neurite growth, differentiation and neuroplasticity (Hancock and Hall, 1993; Liu et al, 2015). 
To be mature and active, Racl and RhoA are subjected to post-translational methylation modification at the C-terminal cysteines, utilizing SAM as a methyl donor cofactor (Roberts et al, 2008). This reaction is catalyzed by isoamyl cysteine carboxyl methyl transferase (LCMT1) (Cushman and Casey, 2009).

Studies have shown that inhibition of LCMT significantly decreases the activation of both RhoA and Rac1, which is due to the increase in RhoGDI $\alpha$ binding by both proteins in the absence of their methylation (Cushman and Casey, 2011). Rac1 and RhoA are activated when they are methylated and bound to GTP, and function in inhibiting neurite growth (Bradke and Dotti, 2000; Liu et al, 2015). RhoGDI $\alpha$ blocks GDP's dissociation from methylated or unmethylated Rac1 and RhoA to keep them inactive, therefore promoting neurite growth (Bradke and Dotti, 2000). Cocaine decreases Racl and RhoA activities and increases dendritic spines in the nucleus accumbens (NAc) (Dietz et al, 2012; Kim et al, 2009). Dopamine inhibits RhoA activity and enhances neurite growth in cultured neurons (Li et al, 2015a). In spite of these advances, the pathway involved in the activation of RhoA and Rac1 is poorly understood.

Dorsal striatum (DS) includes dorsomedial striatum (DMS) and dorsolateral striatum (DLS). DMS primarily regulates goal-directed behavior, whereas the DLS is more involved in habit (Balleine and O'Doherty, 2010). There is a hypothesis that drug addiction is viewed as a transition from voluntary and goal-directed drug use to compulsive habitual drug-seeking. The goal-directed system depends on the interactions between medial prefrontal cortex (mPFC) and DMS (Everitt and Robbins, 2016). In the DMS, dopamine receptor blockade impairs the initial acquisition of goal-directed cocaine seeking (Murray et al, 2012). Moreover, cocaine administration activates extracellular signalregulated kinase signal pathway in the DS that is required for the acquisition of cocaine-induced CPP (Valjent et al, 2006).

CPP is a classical (Pavlovian) assay of drug reward in which acquisition of the preference is measured across multiple conditioning cycles (Mucha et al, 1982). It includes differentially pairing two distinct sets of contextual cues (eg, wall color, pattern) with the stimulus of interest (eg, drug). Conditioning involves animals receiving drug stimulus in one context. Another context without the drug stimulus is control. During a subsequent drug-free CPP test, animals receive unrestricted exposure to both contexts. An increase in time spent is taken as evidence that the drug context was rewarding (Bardo and Bevins, 2000). There are two versions of the CPP termed biased and unbiased. In the biased CPP procedure, drug injections are paired with pretest non-preferred context and saline injections with the pretest preferred context. In the unbiased CPP version the subjects do not show initial side preference and the contexts paired with the drug and saline injections are counterbalanced (Cunningham et al, 2006; Wang, 2003). In the present study, we used a biased CPP procedure to explore the role of NNMT in cocaine reward.

\section{MATERIALS AND METHODS}

\section{Drugs}

Cocaine hydrochloride was purchased from the National Institute for the Control of Pharmaceutical and Biological Products (Beijing, China). MeN was purchased from SigmaAldrich (St Louis, MO, USA). They were dissolved in $0.9 \%$ sterile saline to obtain appropriate concentration for experiment.

\section{Animals}

Adult male C57BL/6J mice, weighing 20 to $22 \mathrm{~g}$, were purchased from Vital River Laboratory Animal Technology Co. Ltd (Beijing, China). All of the animal protocols were reviewed and approved by the Experimental Animal Ethics Committee of Sichuan University (Chengdu, China). Complete description of methods can be found in the Supplementary Methods.

\section{CPP Procedure}

As described previously (Li et al, 2015b), place conditioning studies were conducted utilizing a shuttle box. Complete description of methods can be found in the Supplementary Methods.

\section{Protein Extraction and Western Blot Analysis}

As described previously (Liu et al, 2015; Shang et al, 2016), the protein was extracted by RIPA lysis buffer. Complete description of methods can be found in the Supplementary Methods. The primary antibodies used were as follows: mouse anti-NNMT (29 kDa, 1:500, Abcam), mouse antiRac1 $(21 \mathrm{kDa}, 1: 1000$, Abcam), rabbit anti-RhoA (22 kDa, $1: 1000$, Abcam), rabbit anti-LCMT1 $(38 \mathrm{kDa}, 1: 1000$, Abcam), rabbit anti-RhoGDI $\alpha(23 \mathrm{kDa}, 1: 1000$, Abcam), rabbit anti-B-tubulin ( $50 \mathrm{kDa}, 1: 2000$, ABclonal).

\section{RNA Extraction and PCR Analysis}

As described previously (Shang et al, 2013), the RNA was extracted by Trizol reagent (Invitrogen). Complete description of RNA extraction and PCR analysis can be found in the Supplementary Methods.

\section{Targeted Hydrophilic Interaction Chromatography (HILIC) with Mass Spectrometry}

Metabolites NA, MeN, SAM, and SAH were measured by using tandem mass spectrometry. Complete description of methods can be found in the Supplementary Methods.

\section{Assay for Rac1 and RhoA Activation}

The assay for Rac1 and RhoA activation was performed by using Rac1 and RhoA Activation Assay Kit (NewEast Bioscience), respectively. Complete method is described in the Supplementary Methods. 


\section{Lentivirus Injection}

Four shRNA sequences for silencing Nnmt (ID: 18113) were designed (Supplementary Table S2) by GeneCopoeia (China). Screening tests revealed that No.4 sequence could significantly downregulate the expression of Nnmt mRNA. The target sequence of No.4 was $5^{\prime}$-GAGCAGTTTGAGG TGATTTCT-3'. The lentivirus packaging and mouse treatment are described in the Supplementary Methods.

\section{Co-Immunoprecipitation (Co-IP) Analysis}

We used the commercial co-IP assay kit (Pierce Crosslink Magnetic co-IP Kit, Thermo Scientific) and a RhoGDI $\alpha$ antibody to detect the association of RhoGDI $\alpha$ with Racl or RhoA. Complete description of methods can be found in Supplementary Methods.

\section{Statistical Analysis}

Statistical analysis was performed with IBM SPSS 20.0.0. Statistical analysis was performed by one-way ANOVA for data involving more than two groups. Two groups were analyzed using a Student's $t$-test with a two-tailed $p$-value. Lentivirus or MeN effects on CPP scores were assessed using two-way ANOVAs. All summary statistics of the results are presented as Mean \pm SEM. $p<0.05$ was considered statistically significant.

\section{RESULTS}

\section{Cocaine Upregulates Nnmt Expression in the DS}

To examine the expression of Nnmt in the brain in response to cocaine, mice were conditioned to cocaine in a 6-day CPP protocol (Figure 1a). Mice that received $20 \mathrm{mg} / \mathrm{kg}$ cocaine (i.p.) during conditioning spent significantly more time in the non-preferred chamber during the CPP test, and thereby displayed significantly stronger CPP scores than the mice treated with saline during conditioning (Figure 1b, $\left.t_{(28)}=10.81,{ }^{\star} p<0.0001\right)$. Western blotting showed that NNMT level in the DS was significantly increased in the cocaine-conditioned mice when compared with salineconditioned mice (Figure $1 c, t_{(14)}=3.47,{ }^{\star} p=0.013$ ). However, there were no significant changes in the hippocampus, medial prefrontal cortex (mPFC) and NAc, suggesting a brain region-specific NNMT upregulation in response to cocaine CPP regimen. The brain regions collected for NNMT test were indicated in Supplementary Figure S1A-D. RT-PCR assay showed that Nnmt mRNA level was significantly increased after cocaine CPP procedure in the DS (Supplementary Figure S2A, $\mathrm{t}(12)=3.07,{ }^{\star} p=0.012$ ). To confirm such upregulation was specific to cocaine CPP regimen, we compared the NNMT level in the DS of mice $30 \mathrm{~min}$ and $24 \mathrm{~h}$ after single cocaine injection $(20 \mathrm{mg} / \mathrm{kg}$, i.p.) and after 7-day repeated cocaine treatment $(20 \mathrm{mg} / \mathrm{kg}$ per day, i.p.), respectively. NNMT protein level has no significant change $30 \mathrm{~min}$ or $24 \mathrm{~h}$ after single cocaine challenge. However, 7-day repeated cocaine treatment upregulated NNMT protein level (Figure 1d, $t_{(14)}=3.93$, $\left.{ }^{*} p=0.0077\right)$. These results showed that chronic cocaine induces NNMT upregulation in the DS.
Cocaine Decreases the Levels of SAM/SAH Ratio in the DS

To analyze the levels of NNMT-related metabolites in the DS, targeted HILIC-QTOF mass spectrometry was used to separate and identify the metabolites, including SAM and NA, the substrates of NNMT, as well as SAH and MeN, the products of NNMT. Extracted ion chromatogram (EIC) of these 4 metabolites were shown in the Supplementary Figure S3. Retention times and major adducts for each compound are as follows: MeN $(m / z$ 137.0715) $2.44 \mathrm{~min}, \mathrm{NA}$ $(m / z$ 123.0558) $0.88 \mathrm{~min} \mathrm{M}+\mathrm{H}, \mathrm{SAH}(\mathrm{m} / z 385.1294) 3.05 \mathrm{~min}$ $\mathrm{M}+\mathrm{H}$, SAM $(\mathrm{m} / z$ 399.1451) $3.38 \mathrm{~min}$. The result indicated that SAM/SAH ratio levels in the DS was significantly reduced in cocaine-conditioned mice (Figure 1e, $t_{(18)}=3.60$, $\left.{ }^{*} p=0.0032\right)$, whereas MeN/NA levels were increased (Figure 1f, $\left.t_{(18)}=3.48,{ }^{*} p=0.0040\right)$. These results were consistent with the aforementioned finding that Nnmt expression was upregulated by cocaine, suggesting that cocaine can upregulate the level and activity of NNMT, leading to the alteration of SAM/SAH ratio levels.

\section{Cocaine Decreases the Activities of Racl and RhoA in the DS}

To be active, Racl and RhoA are subjected to posttranslational methylation modification, utilizing SAM as a cofactor(Roberts et al, 2008). We first investigated whether the activities of Racl and RhoA could be modified by cocaine. Configuration-specific monoclonal antibodies that specifically recognize Rac1-GTP or RhoA-GTP, but not Rac1-GDP or RhoA-GDP, were used for immunoprecipitation assay. We found that the activities of $\operatorname{Rac} 1\left(t_{(14)}=11.55\right.$, $\left.{ }^{\star} p=0.0003\right)$ and RhoA $\left(t_{(14)}=4.41,{ }^{\star} p=0.012\right)$ were significantly reduced in the DS of cocaine-conditioned mice (Figure 1g, i, $j$ and 1 ). There was no significant change in the protein level of Racl (Figure 1h and i); however, RhoA was upregulated (Figure $1 \mathrm{k}$ and $\mathrm{l} ; t_{(14)}=4.88,{ }^{\star} p=0.0028$ ). We continued to use RT-PCR to detect the mRNA level of Rac1 and $R h o A$. We found that there was no significant change in Rac1 mRNA level in the DS of cocaine-conditioned mice (Supplementary Figure S2B), whereas cocaine significantly elevated the transcription of RhoA gene (Supplementary Figure S2C, $\left.\mathrm{t}(12)=4.33,{ }^{\star} p=0.0019\right)$. These results indicated that cocaine could attenuate Racl activity but without affecting the transcription and translation of Racl gene. In spite that cocaine elevated $R h o A$ expression, but its activity was decreased.

\section{Nnmt Knockdown in the DMS Decreases Cocaine CPP}

To test the effect of NNMT on cocaine effect in vivo, we knocked down Nnmt in the dorsomedial striatum (DMS) of C57BL6/J mice by using lentivirus (LV). Mice were received bilateral intra-DMS injection of LV expressing either scrambled shRNA (LV-NC) or Nnmt interference sequence (LV-Sh-Nnmt). The coordinate of lentivirus injection site was shown in Figure 2a. After recovery, the mice were subjected to cocaine CPP procedure (Figure $2 \mathrm{~b}$ ). As shown in the Figure $2 \mathrm{a}$, the lentivirus eGFP vector was successfully infected and expressed in the DMS, indicating correct coordinates for intracranial injection. We then detected 
Nnmt expression to confirm the silencing effect of LV-ShNnmt. As expected, Nnmt expression in the DMS receiving LV-Sh-Nnmt was significantly decreased as compared with that receiving LV-NC (mRNA: Figure $2 c, F_{(3,24)}=19.17$, $\mathrm{COC}+\mathrm{LV}-\mathrm{NC}$ vs SAL+LV-NC ${ }^{*} p<0.0001$, COC+LV-ShNnmt vs COC+LV-NC $\# p=0.0004$, SAL+LV-Sh-Nnmt vs a

SAL

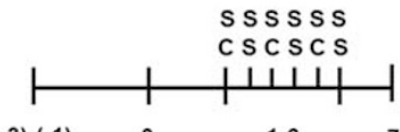

Days $\begin{array}{llll}(-3)-(-1) & 0 & 1-6 & 7\end{array}$ Handling Baseline CPPTraining Test

b

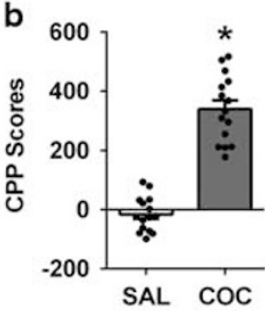

C
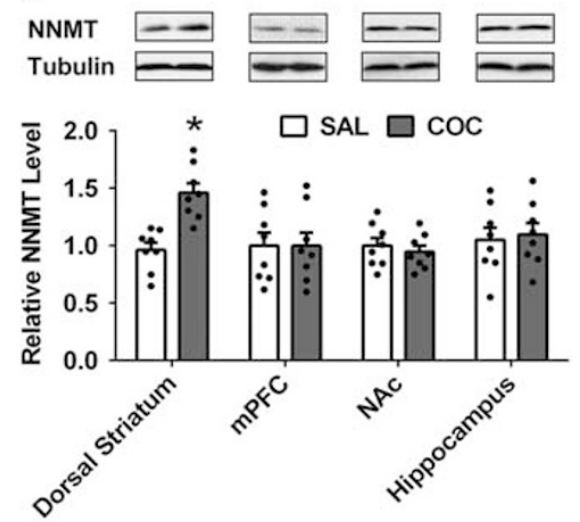

d

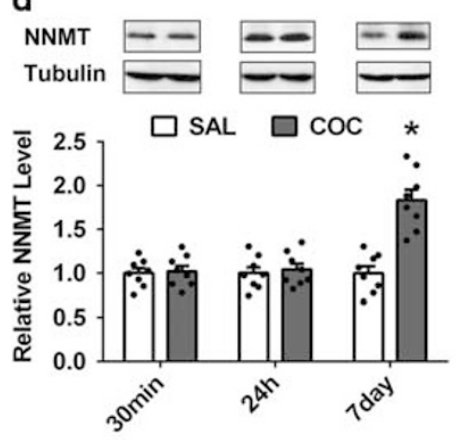

g

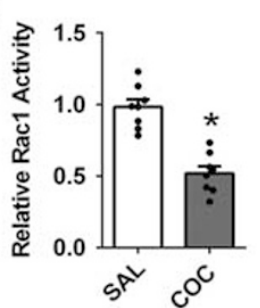

j

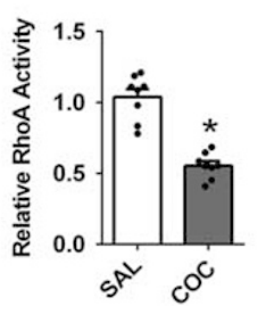

e

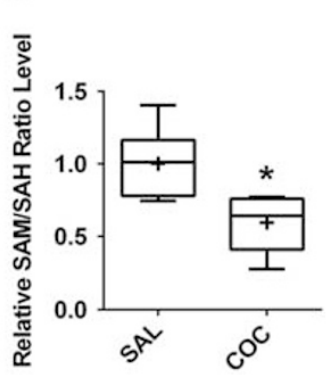

h

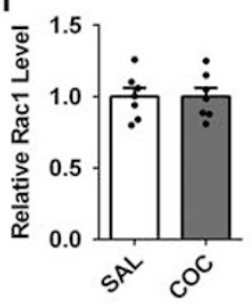

$\mathbf{k}$

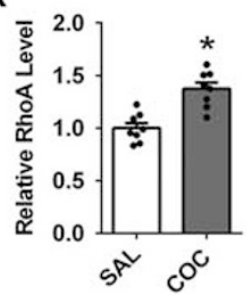

$\mathbf{f}$

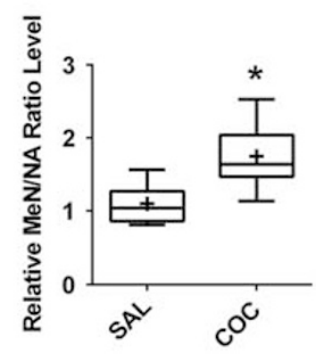

i

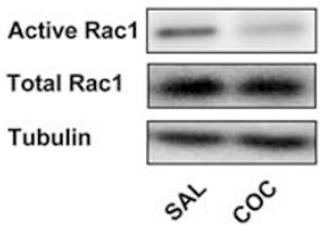

I

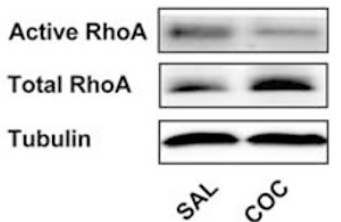

Figure I Cocaine upregulates NNMT and reduces the activities of Racl and RhoA in the DS. (a) Timeline of cocaine CPP experiment. After test, mice brain tissues were harvested for molecular experiment. (b) CPP score of cocaine group $(20 \mathrm{mg} / \mathrm{kg}$ i.p.) was significantly higher than that of saline group. $n=15$ per group. (c) Western blotting analysis showed that NNMT level in cocaine group was higher than that of saline group in the DS, but not in the mPFC, NAc, and hippocampus. $n=8$ per group. (d) Acute cocaine did not alter the expression of Nnmt in the DS. Repeated cocaine exposure induced upregulation of NNMT in the DS as compared to saline treatment. $n=8$ per group. (e) Box-and-whisker plots illustrated the changes of SAM/SAH ratio levels in saline and cocaine groups, respectively. SAM/SAH ratio levels were decreased by cocaine. $n=10$ per group. (f) MeN/NA ratio levels were increased by cocaine. $n=10$ per group. (g) Racl activity in cocaine group was lower than that of saline group. Active Racl normalized to total Racl. $n=8$ per group. (h) Western blotting analysis indicated that total Racl level showed no significant difference between cocaine and saline group. Total Racl normalized to $\beta$-tubulin. $n=8$ per group. (i) Immunoblotting of Racl and $\beta$-tubulin. (j) The activity of RhoA in cocaine group was lower than that of saline group. $n=8$ per group. ( $k$ ) Western blotting analysis indicated that total RhoA level in cocaine group was higher than that of saline group. $n=8$ per group. (I) Immunoblotting of RhoA and $\beta$-tubulin. *, compared with SAL group; C, cocaine; COC, intraperitoneal injection of cocaine; CPP, conditioned place preference; MeN, I-methylnicotinamide; mPFC, medial prefrontal cortex; NA, nicotinamide; NAc, nucleus accumbens; S, saline; SAH, S-adenosyl-L-homocysteine; SAL, intraperitoneal injection of saline; SAM, S-adenosyl-L-methionine. 
a
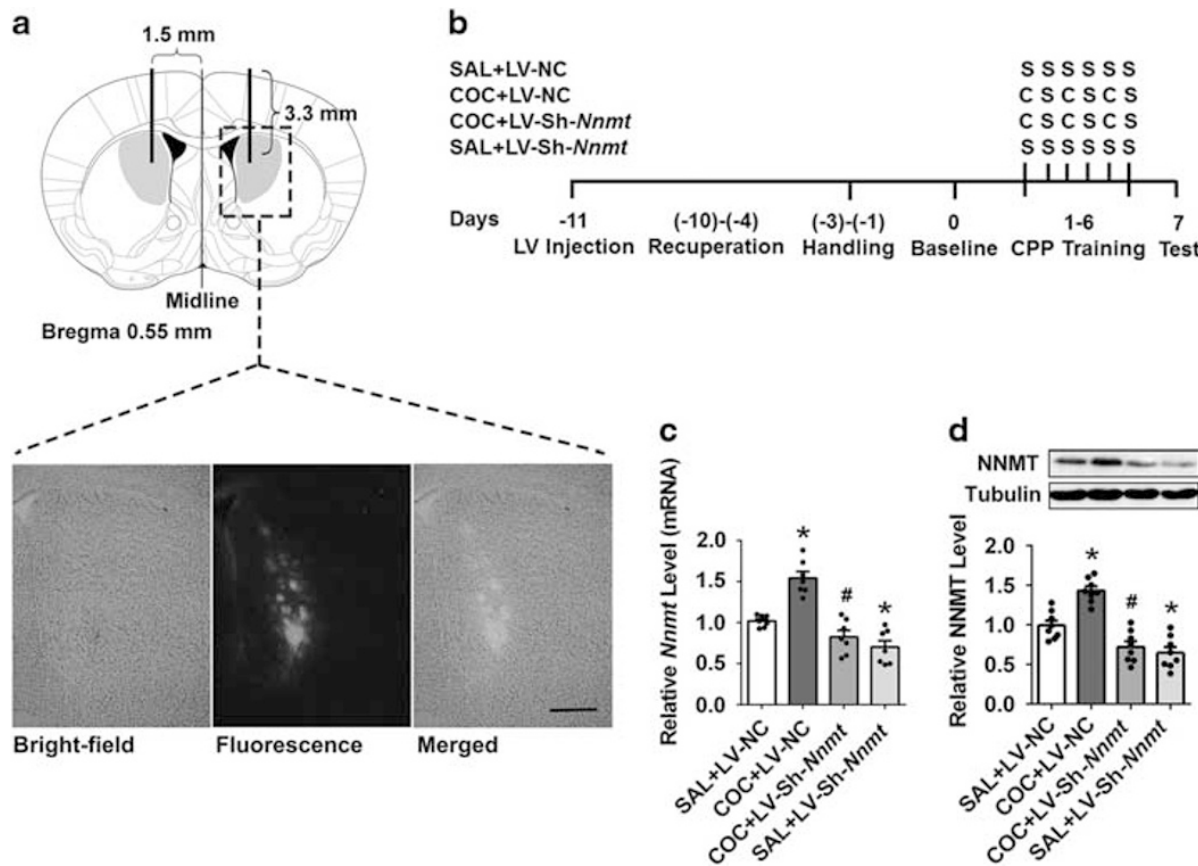

Figure 2 Nnmt-shRNA lentivirus decreases Nnmt expression in the DMS. (a) Brain atlas showed the lentivirus injection sites in the DMS (blue area). Lentivirus vectors expressing eGFP are successfully transfected and expressed in the DMS. Bar $=400 \mu \mathrm{m}$. (b) Before cocaine CPP experiment, mice were subjected to lentivirus injection to knockdown Nnmt expression in the DMS. After test, mice brain tissues were harvested for molecular experiment. (c) RT-PCR analysis indicated that Nnmt-shRNA lentivirus significantly decreased Nnmt transcription in the DMS. SAL+LV-Sh-Nnmt compared with SAL+LVNC; and COC+LV-Sh-Nnmt compared with COC+LV-NC. $n=7$ per group. (d) Western blotting analysis showed that the NNMT level in the DMS receiving LV-Sh-Nnmt was decreased significantly as compared with that receiving LV-NC. $n=8$ per group. *, compared with SAL+LV-NC group; \#, compared with COC+LV-NC group; DMS, dorsomedial striatum; C, cocaine; S, saline; COC, intraperitoneal injection of cocaine; SAL, intraperitoneal injection of saline; LV-NC, intra-DMS injection of negative control lentivirus expressing scrambled shRNA; LV-Sh-Nnmt, intra-DMS injection of lentivirus expressing Nnmt shRNA; CPP, conditioned place preference. A full color version of this figure is available at the Neuropsychopharmacology journal online.

SAL $+\mathrm{LV}-\mathrm{NC}{ }^{*} p=0.0024$; and protein: Figure $2 \mathrm{~d}, F_{(3,28)}$ $=23.17, \mathrm{COC}+\mathrm{LV}-\mathrm{NC}$ vs SAL+LV-NC ${ }^{*} p=0.0014, \mathrm{COC}$ $+\mathrm{LV}-\mathrm{Sh}-\mathrm{Nnm} t$ vs COC+LV-NC $\# p=0.0011$, SAL+LV-ShNnmt vs SAL+LV-NC $\left.{ }^{*} p=0.047\right)$. In CPP experiment, a two-way ANOVA revealed the significant main effects of cocaine $\quad\left(F_{(1,56)}=181.10, \quad p<0.0001\right)$ and lentivirus $\left(F_{(1,56)}=9.19, p=0.0037\right)$, as well as cocaine $\times$ lentivirus interactions $\left(F_{(1,56)}=15.22, p=0.0003\right)$. Through post hoc test (Tukey's multiple comparisons) we observed that the cocaine-conditioned mice treated with LV-Sh-Nnmt showed significantly decreased CPP scores than the control mice treated with LV-NC (Figure 3a, $q_{(4,56)}=6.93$, $\# p<0.0001$ ). Collectively, these results indicated that Nnmt knockdown in the DMS could significantly attenuate cocaine effect.

\section{NNMT Regulates Cocaine CPP Possibly though SAM- Rac1/RhoA Pathway}

To examine whether Nnmt knockdown would modify the ratio levels of SAM/SAH in the DMS, targeted HILIC-QTOF mass spectrometry was used to measure the levels of the metabolites. The results showed that cocaine significantly reduced the levels of SAM/SAH ratio in the DMS, whereas such decreases were inhibited by Nnmt knockdown (Figure $3 b, F_{(2,27)}=6.46,{ }^{*} p=0.0043, \# p=0.015$ ). Conversely, cocaine significantly elevated $\mathrm{MeN} / \mathrm{NA}$ ratio levels, whereas such increases were inhibited by Nnmt knockdown (Figure 3c, $F_{(2,27)}=14.53,{ }^{\star} p=0.0007, \#^{*} p=0.0044$ ).
We next tested whether NNMT-induced changes in SAM/ $\mathrm{SAH}$ ratio could affect the activity of protein through methylation events. We measured the activities of Rac1 and RhoA with or without Nnmt knockdown in the DMS. Importantly, we found that cocaine reduced the activity of Rac1 and RhoA, whereas such decreases were inhibited by Nnmt knockdown (Rac1: Figure $3 \mathrm{~d}$ and $\mathrm{h} ; F_{(2,21)}=37.22$, ${ }^{*} p=0.0016, \# p=0.0025$; RhoA: Figure $3 \mathrm{f}$ and $i ; F_{(2,21)}=7.44$, $\left.{ }^{*} p=0.020,{ }^{*} p=0.016\right)$. These results revealed the involvement of NNMT in regulating the activities of Racl and RhoA. We continued to confirm the protein level of total Rac1 and RhoA. There was no significant change of Rac1 after Nnmt knockdown (Figure 3e and h); however, RhoA was downregulated (Figure $3 g$ and i; $F_{(2,21)}=4.59$, $\left.{ }^{*} p=0.011, \# p=0.048\right)$. The RT-PCR results further showed that there were no significant changes in the mRNA level of Rac1 in the DMS (Supplementary Figure S4A), whereas Nnmt downregulation inhibited the transcription of RhoA gene (Supplementary Figure S4B, $F_{(3,24)}=7.59$, COC+LV$\mathrm{NC}$ vs SAL+LV-NC ${ }^{*} p=0.0011, \mathrm{COC}+\mathrm{LV}-\mathrm{Sh}-N n m t$ vs COC $+\mathrm{LV}-\mathrm{NC} \quad \# p=0.046$, SAL+LV-Sh-Nnmt vs SAL+LV-NC $\left.{ }^{*} p=0.043\right)$. Collectively, these results indicated that Nnmt knockdown can reverse cocaine-induced downregulation of Rac1 and RhoA activities.

As post-translational methylation modification of Rac1 and RhoA is catalyzed by LCMT1 (Cushman and Casey, 2009), we next investigated whether LCMT1 could be involved in the regulation of NNMT on Rac1 and RhoA. 
a

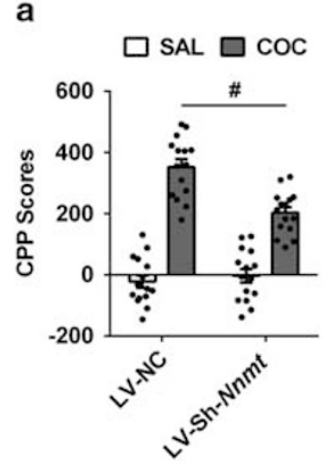

b

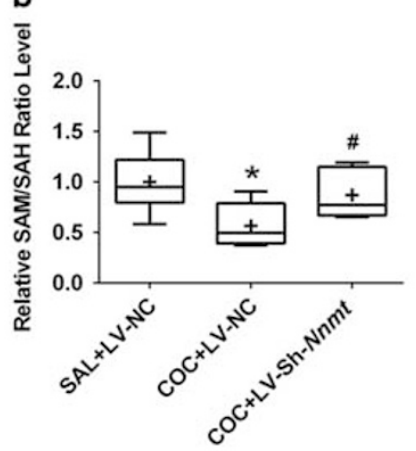

c

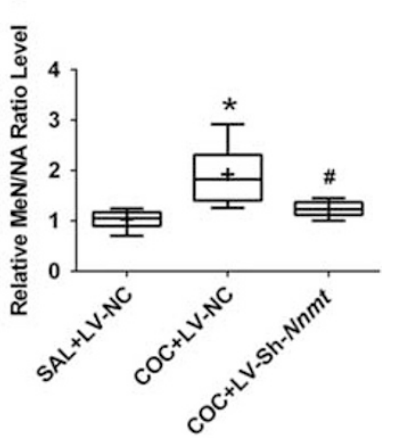

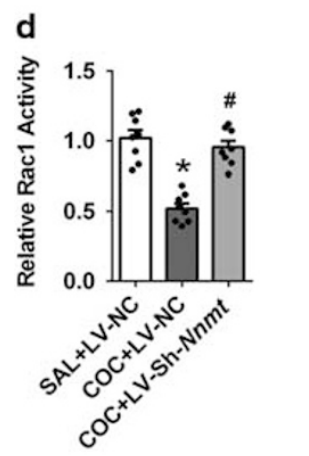

e

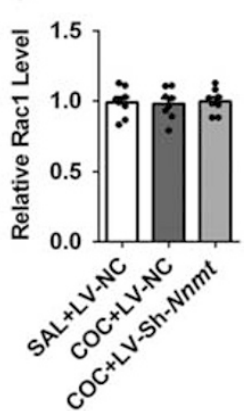

f

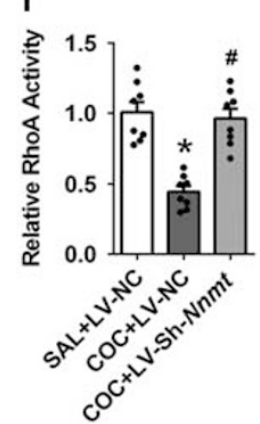

g

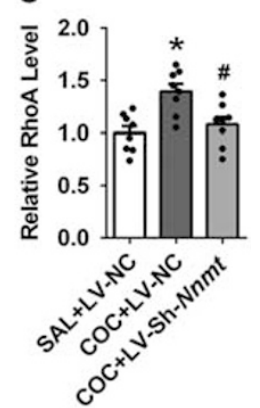

h

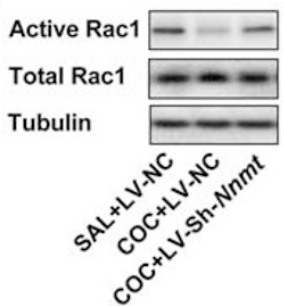

i

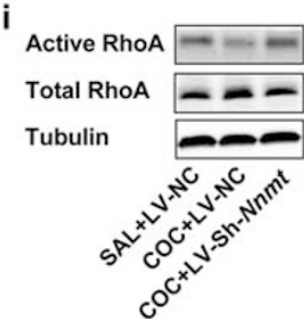

Figure 3 Nnmt knockdown in the DMS decreases cocaine CPP and increases the activities of Racl and RhoA. (a) Nnmt knockdown in the DMS weakened cocaine-induced CPP. $n=15$ per group. (b) For more clearly showing the effect of lentivirus in cocaine-treated mice, SAL+LV-Sh-Nnmt group in subsequent tests was not shown. Box-and-whisker plots illustrated the SAM/SAH ratio levels were decreased by cocaine, but increased by Nnmt knockdown. $n=10$ per group. (c) MeN/NA ratio levels were increased by cocaine, but reduced by Nnmt knockdown. $n=10$ per group. (d) Racl activity was reduced by cocaine, but increased by Nnmt knockdown. Active Racl normalized to total Racl. $n=8$ per group. (e) The level of total Racl protein showed no difference after COC + LV-NC-treated or COC+LV-Sh-Nnmt-treated in the DMS. Total Racl normalized to $\beta$-tubulin. $n=8$ per group. (f) RhoA activity was also reduced by cocaine, but increased by Nnmt knockdown. $n=8$ per group. (g) The level of total RhoA protein increased after COC+LV-NC treatment, but decreased after COC+LV-Sh-Nnmt treatment. $n=8$ per group. (h) Immunoblotting of Racl and $\beta$-tubulin. (i) Immunoblotting of RhoA and $\beta$-tubulin. *, compared with SAL +LV-NC group; \#, compared with COC+LV-NC group; DMS, dorsomedial striatum; COC, intraperitoneal injection of cocaine; SAL, intraperitoneal injection of saline; LV-NC, intra-DMS injection of negative control lentivirus expressing scrambled shRNA; LV-Sh-Nnmt, intra-DMS injection of lentivirus expressing Nnmt shRNA; CPP, conditioned place preference. SAM, S-adenosyl-L-methionine; SAH, S-adenosyl-L-homocysteine; MeN, I-methylnicotinamide; NA, nicotinamide.

By using RT-PCR and western blotting, we measured the expressions of Lcmt1. However, there was no significant change in Lcmt1 expression in cocaine-conditioned mice with or without LV-Sh-Nnmt treatment (Supplementary Figure S4C and D). Therefore, LCMT1 may not mediate the effect of NNMT on the methylation modification of Rac1 and RhoA. As maturation and activation of Racl and RhoA rely on post-translational methylation, we consider that Nnmt knockdown activates Racl and RhoA possibly through elevating SAM/SAH ratio levels in the DMS of mice treated with cocaine. Elevated SAM/SAH levels account for, at least in part, the inhibitory effect of Nnmt knockdown on cocaine effect.

\section{Nnmt Knockdown Inhibits the Association of RhoGDI $\alpha$ with Racl or RhoA in the DMS}

As RhoGDI $\alpha$ plays an important role in GDP binding to Racl or RhoA, we detected the RhoGDI $\alpha$ level in extracted total protein (Input protein). We observed that there was no significant change in the RhoGDI $\alpha$ translation level of cocaine-conditioned mice with or without Nnmt silence (Figure 4a and e). To explore whether RhoGDI $\alpha$ could mediate the effect of NNMT on Racl and RhoA activities, co-IP analysis using a RhoGDI $\alpha$ antibody was performed to detect the association of RhoGDI $\alpha$ with Racl or RhoA. Importantly, we found that cocaine increased the interaction of RhoGDI $\alpha$ with Racl or RhoA in the DMS, whereas such 

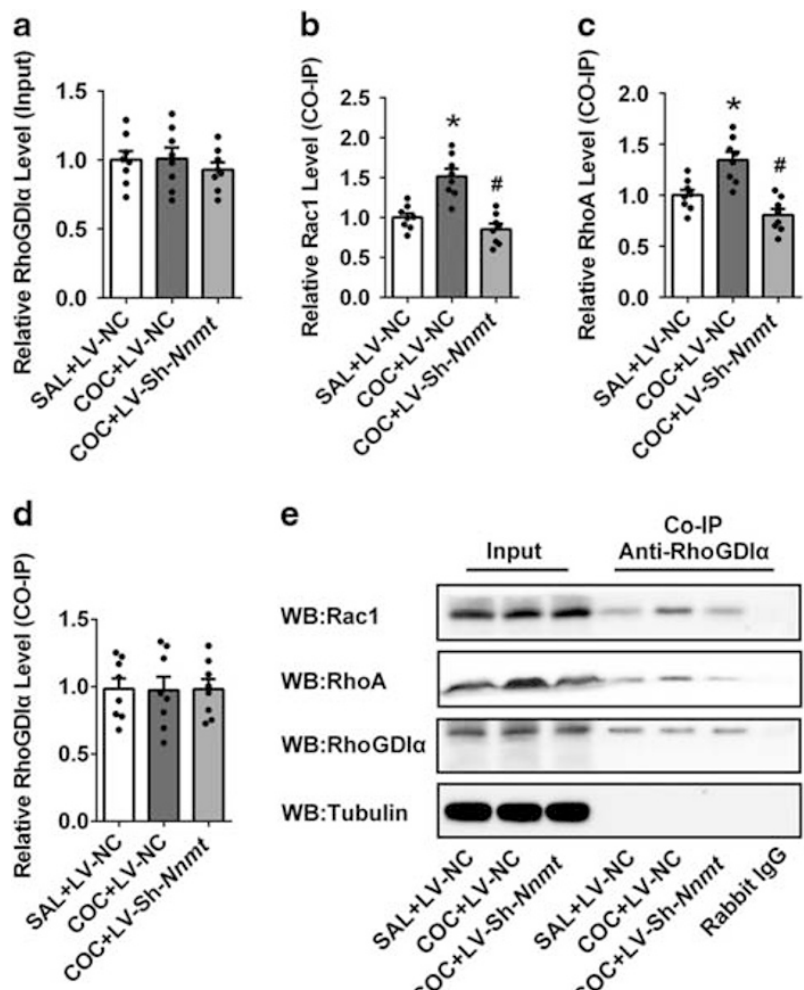

e

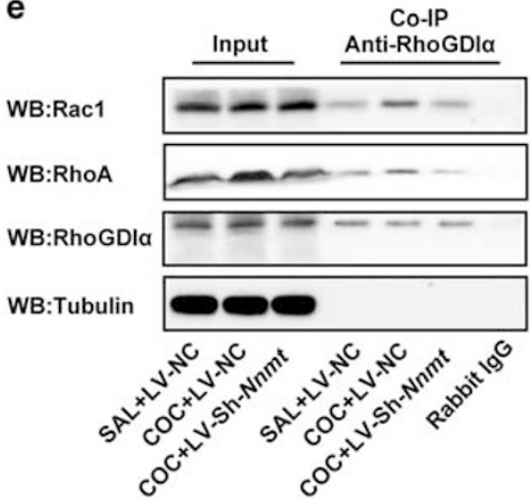

Figure 4 Nnmt knockdown in the DMS inhibits the interaction of RhoGDl $\alpha$ with Racl or RhoA. (a) RhoGDl $\alpha$ protein level showed no difference between saline and cocaine group in the DMS. Nnmt knockdown did not influence RhoGDl $\alpha$ translation. Total input RhoGDl $\alpha$ normalized to $\beta$-tubulin. $n=8$ per group. (b) The level of Racl enriched by a RhoGDl $\alpha$ antibody in the DMS was increased by cocaine, but reduced by Nnmt knockdown. Co-IP Racl normalized to input Racl. $n=8$ per group. (c) The level of RhoA enriched by a RhoGDl $\alpha$ antibody in the DMS was also increased by cocaine, but reduced by Nnmt knockdown. $n=8$ per group. (d) The level of RhoGDl $\alpha$ enriched by a RhoGDl $\alpha$ antibody in the DMS showed no change by cocaine or by Nnmt knockdown. Co-IP RhoGDl $\alpha$ normalized to input RhoGDl $\alpha . n=8$ per group. (e) Co-IP analysis with a RhoGDla antibody. *, compared with SAL+LV-NC group; \#, compared with COC+LV-NC group; Rabbit IgG, a negative control; Input, total protein before co-IP; COC, intraperitoneal injection of cocaine; SAL, intraperitoneal injection of saline; LV-NC, intra-DMS injection of negative control lentivirus expressing scrambled shRNA; LV-Sh-Nnmt, intra-DMS injection of lentivirus expressing Nnmt shRNA.

increases were blocked by Nnmt knockdown through an infusion of LV-Sh-Nnmt (Racl: Figure $4 \mathrm{~b}$ and e; $F_{(2}$, 21) $=9.05,{ }^{*} p=0.023, \# p=0.012$; and RhoA: Figure $4 c$ and e; $\left.F_{(2,21)}=7.51,{ }^{\star} p=0.028, \# p=0.015\right)$. We also detected the level of RhoGDI $\alpha$ that was immuno-precipitated by RhoG$\mathrm{DI} \alpha$ antibody. However, there was no significant change in the RhoGDI $\alpha$ level of cocaine-conditioned mice with or without Nnmt silence (Figure $4 \mathrm{~d}$ and e). These results showed that the dynamic interaction of RhoGDI $\alpha$ with Rac1 or RhoA, rather than RhoGDI $\alpha$ translation, may mediate the effect of NNMT in regulating cocaine effect.

\section{Pharmacological Inhibition of NNMT in the DMS Inhibits Cocaine CPP and Racl and RhoA Activities}

$\mathrm{MeN}$, a reaction product of NNMT, is a potent inhibitor of NNMT through a feedback mechanism (Kraus et al, 2014).
To examine whether the effect of NNMT on cocaine CPP would be mediated by its metabolic product, the DMS of mice was intracerebrally infused with $\mathrm{MeN}$ solution $(2,10$, and $50 \mathrm{mM}$, respectively) at a volume of $0.5 \mu \mathrm{l}$ per brain side (Figure 5a). The intracerebral injection was $30 \mathrm{~min}$ prior to cocaine injection, and then behavioral training was conducted and cocaine CPP was measured (Figure 5b). Two-way ANOVA revealed the significant main effects of cocaine $\left.\left(F_{(1,} 104\right)=352.00, p<0.0001\right)$ and a significant cocaine $\times \mathrm{MeN}$ interactions $\left(F_{(3,104)}=3.60\right.$, $P=0.0465)$; however, no significant main effect of $\mathrm{MeN}$ was observed $\left(F_{(3,104)}=2.493, p=0.0641\right)$. Through post-hoc test (Tukey's multiple comparisons), we found that the cocaine-conditioned mice treated with $50 \mathrm{mM}$ MeN showed significantly decreased CPP scores than the control mice treated with saline (NC) (Figure 5c, $q_{(8,104)}=5.152$, $\# p<0.01)$. However, $\mathrm{MeN}$ at the dose of $2 \mathrm{mM}$ or $10 \mathrm{mM}$ showed no obvious effect. In order to confirm the inhibitory action of MeN on NNMT activity, we used targeted HILICQTOF mass spectrometry to measure the metabolites. The results showed that intra-DMS injections of $50 \mathrm{mM} \mathrm{MeN}$ significantly elevated SAM/SAH ratio (Figure $5 \mathrm{~d}, t_{(18)}=4.51$, $\left.{ }^{\#} p=0.0006\right)$. Elevated $\mathrm{MeN} / \mathrm{NA}$ ratio indicated a successful infusion of $\mathrm{MeN}$ into the DMS (Figure 5e, $t_{(18)}=8.07$, $\# p<0.0001)$. We continued to measure the activities of Rac1 and RhoA in the DMS, and found that MeN increased the activities of these two molecules (Rac1: Figure $5 \mathrm{f}$ and h; $t_{(14)}=3.31, \# p=0.016$; RhoA: Figure $5 \mathrm{~g}$ and $\mathrm{j} ; t_{(14)}=8.13$, $\# p=0.0002)$. The total protein level of Rac1 was not significantly changed (Figure $5 \mathrm{f}$ and $\mathrm{i}$ ), whereas RhoA was downregulated by $50 \mathrm{mM} \mathrm{MeN}$ (Figure $5 \mathrm{~g}$ and $\mathrm{k} ; t_{(14)}=3.13$, $\left.{ }^{\#} P=0.021\right)$.

\section{DISCUSSION}

Altered methyl supply with an increase in brain SAM is associated with methyltransferase level and becomes a crucial factor mediating brain function (Jayaram et al, 2016; Saunderson et al, 2016). NNMT is an important enzyme of one carbon metabolism, using SAM as its cofactor. The roles of NNMT in liver metabolism and cancer have been investigated (Hong et al, 2015; Xu et al, 2016); however, the role of NNMT in drug addiction is largely unknown. Here, we found NNMT decreases SAM level in the DMS of cocaine-conditioned mice, which is accompanied by the reduced post-translational methylation of Racl and RhoA. Unmethylated Rac1/RhoA combine with GDP to form inactive Rac1 GDP/RhoA GDP, which interact with RhoGDI $\alpha$ to decrease their function in inhibiting neurite growth. These findings showed that NNMT regulates cocaine CPP possibly through SAM-mediated regulation of Racland RhoA activities (Figure 5l).

\section{Methyl-Modification of DNA, Histone and Nonhistone Protein Play Roles in Cocaine CPP}

SAM supplies methyl for many biochemical reactions in vivo (Zhang and Zheng, 2016). In fact, enhancing the pool of available methyl donors with SAM or its precursor increases DNA methylation and alters gene expression. In drug addiction, previous studies most focused on the relationship 


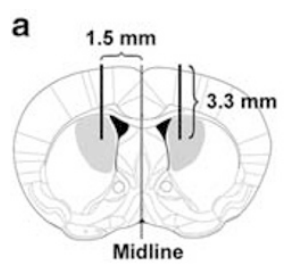

Bregma $0.55 \mathrm{~mm}$

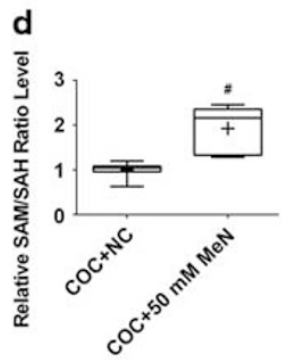

h

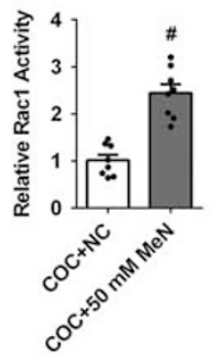

j

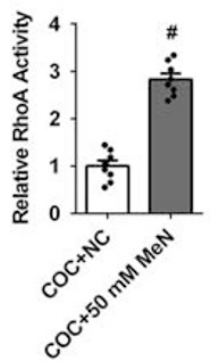

b

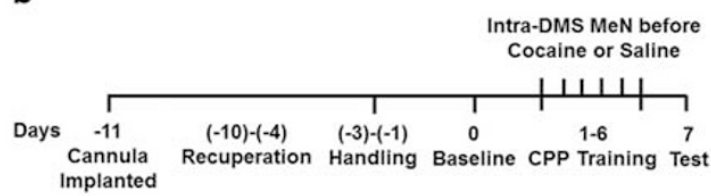

e

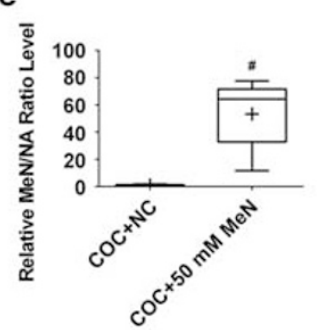

i

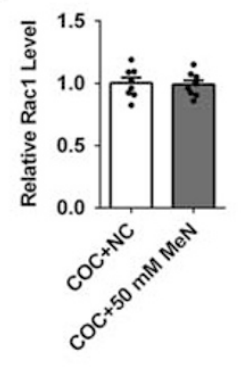

k

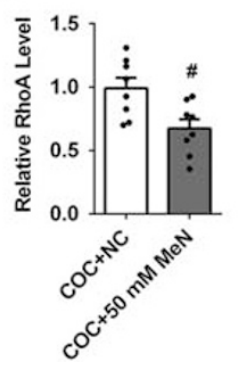

f

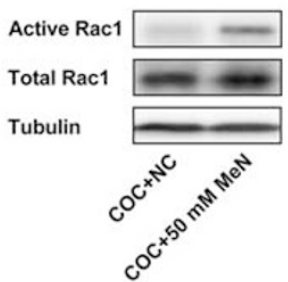

I

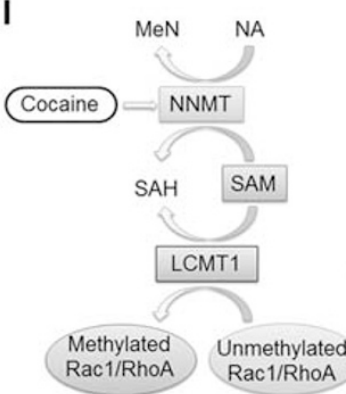

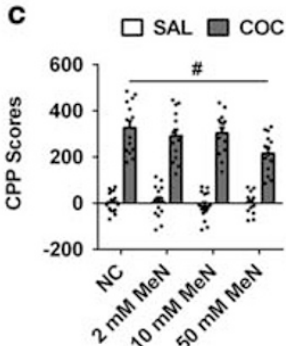

g

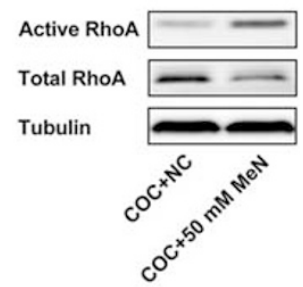

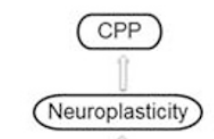

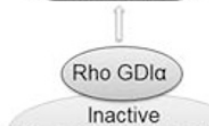

Rac1 GDP/RhoA GDP

Figure 5 MeN regulate cocaine CPP though Racl and RhoA. (a) Brain atlas showed MeN injection sites in the DMS (blue areas). (b) Before cocaine CPP training, mice were subjected to intra-DMS infusion of MeN to inhibit NNMT. (c) Intra-DMS injection of 50 mM MeN decreased cocaine-induced CPP. $n=14$ per group. (d) SAM/SAH ratio were increased by Intra-DMS injection of $50 \mathrm{mM} \mathrm{MeN.} n=10$ per group. (e) MeN/NA ratio were increased by intra-DMS injection of $50 \mathrm{mM}$ MeN. $n=10$, per group. (f) Immunoblotting of Racl and $\beta$-tubulin. (g) Immunoblotting of RhoA and $\beta$-tubulin. (h) Racl activity was increased by intra-DMS injection of $50 \mathrm{mMMeN}$. Active Racl normalized to total Racl. $n=8$ per group. (i) Racl protein level showed no difference between $\mathrm{COC}+\mathrm{NC}$ and $\mathrm{COC}+50 \mathrm{mM}$ MeN groups in the DMS. Total Racl normalized to $\beta$-tubulin. $n=8$ per group. (j) RhoA activity was increased by $50 \mathrm{mM}$ MeN. $n=8$ per group. ( $k$ ) RhoA protein level was decreased by intra-DMS injection of $50 \mathrm{mM} \mathrm{MeN.} n=8$ per group. (I) Schematic representation of putative mechanism of NNMT in cocaine-induced CPP. Yellow: upregulation; blue: downregulation; gray: unchanged. \#p, compared with COC+NC group; CPP, conditioned place preference; SAL, intraperitoneal injection of saline; COC, intraperitoneal injection of cocaine; NC, intra-DMS injection of saline; MeN, intra-DMS injection of MeN; SAM, S-adenosyl-L-methionine; SAH, S-adenosyl-L-homocysteine; MeN, I-methylnicotinamide; NA, nicotinamide. A full color version of this figure is available at the Neuropsychopharmacology journal online.

between SAM and the methylation of DNA or histone (Anier et al, 2010; Black et al, 2006; Jordi et al, 2013; Maze and Nestler, 2011). In addition, there exists some methylation of nonhistone protein assisted by SAM (Aksoy et al, 1995). Racl and RhoA, two important members of Rho GTPase family, require post-translational methylation using SAM as the methyl donor ( $\mathrm{Li}$ et al, 2015a). Rac1 and RhoA are effectors of D1 receptor signaling during dendritic morphogenesis in the PFC ( $\mathrm{Li}$ et al, 2015a). Blocking Racl activity increases the density of immature dendritic spines of neurons in the NAc (Dietz et al, 2012). However, the mechanism underlying the activation of Racl and RhoA is still largely unknown. In the present study, we found that cocaine reduced SAM/SAH ratio levels as well as Rac1 and RhoA activities in the DS, which may be due to the incomplete post-translational methylation as a result of the reduced supply of SAM. 
Deficient Methyl-Modification of Rac1 and RhoA may be the Result of Decreased SAM in Cocaine-Conditioned Mice

After binding to GTP, Rho GTPase family becomes activated Rho GTP; on the contrary, after binding to GDP, Rho GTPase family becomes inactivated Rho GDP (Jaffe and Hall, 2005; Tiedje et al, 2008). RhoGDI $\alpha$ blocks the dissociation of GDP from Racl and RhoA, making them inactive, which enhances the neurite growth, differentiation and neuroplasticity (Hancock and Hall, 1993; Liu et al, 2015). It is known that decreased methylation of Racl and RhoA increases their binding to RhoGDI $\alpha$ (Cushman and Casey, 2011). We found that cocaine induced a decrease in SAM but an increase in the interaction of RhoGDI $\alpha$ with Rac1 and RhoA in the DMS. However, such effect could be reversed by Nnmt knockdown. Therefore, these results suggest that the incomplete post-translational methylations of Racl and RhoA may be the result of decreased SAM in the DMS of cocaine-conditioned mice. The exact mechanism by which SAM regulates the methylation of Racl and RhoA needs to be addressed in the future.

\section{LCMT1 may not Participate in the Regulation of Rac1 and RhoA Activities after Cocaine Exposure}

RhoA and Racl are best known as critical regulators of cytoskeleton dynamics and neuronal development. Posttranslational methylation of Racl and RhoA are catalyzed by LCMT1 (Nishimura and Linder, 2013). It has been known that inhibition of LCMT1 decreases the methylation levels of Racl and RhoA, thus weakening the activities of Racl and RhoA (Cushman and Casey, 2009). However, in this study, cocaine treatment or Nnmt knockdown had no effect on Lcmt1 expression. So far, there is no evidence showing a post-translational modification that influences LCMT1 activity. Our results might exclude the involvement of LCMT1 in regulating Rac1 and RhoA activities.

\section{DNA Methylation may Contribute to the Discordance of RhoA Expression}

We found that NNMT regulated RhoA expression in the DMS of cocaine-conditioned mice, which was accompanied by the altered SAM level. Moreover, RhoA was upregulated or downregulated along with SAM depletion or elevation. It is known that SAM supplies methyl for DNA methylation (Jones and Takai, 2001; Shang et al, 2016). Generally, DNA hypomethylation changes protein-DNA interactions, leading to the alterations in chromatin structure and transcription rate, which mainly promotes gene expression. On the other hand, DNA hypermethylation inhibits gene expression (Jones and Takai, 2001; Zhang and Zheng, 2016). Methylated $\mathrm{CpG}$ island regions of DNA exist in the mouse promoter region of RhoA gene (NCBI Gene database, NC_000075.6). Considering above, we infer that SAM supply may also influence DNA methylation of RhoA gene and regulate its expression responding to cocaine.
Post-translational Methylation is not Necessary for CDC42

Besides Rac1 and RhoA, CDC42 is another important member of Rho GTPase family (Li et al, 2002). In the present study, we did not focus on $\mathrm{CDC} 42$ because its posttranslational modification is different from Racl and RhoA. After translation, the C-terminal of Racl and RhoA exposes CAAX sequences. The cysteine is isoprentenylated, and AAX peptide is hydrolyzed. The exposed cysteine is then methylated by LCMT1. In this process, SAM provides methyl. However, the $\mathrm{C}$ terminal amino sequence of CDC42 is CCIF. Although cysteine is isoprentenylated, the peptide is not hydrolyzed and does not expose cysteine (Nishimura and Linder, 2013). These reports indicate that post-translational methylation and SAM are not necessary for CDC42. Similarly, a previous study showed the activities of Racl and RhoA are decreased in the NAc of cocaine-treated mice, but CDC42 activity is not changed (Dietz et al, 2012).

\section{Comparison of Biased and Unbiased Design of CPP}

There are two different designs for CPP test, biased and unbiased designs. In biased design, mice show a clear preference for one chamber. Cocaine is paired with the less preferred chamber to reverse mice preference. In unbiased design, mice are assigned randomly. Generally, biased design is considered when the average time that untrained animals spend in each test chamber deviates from expectations based on chance. Conversely, unbiased design is considered when the time is consistent with expectations based on chance (eg, average of $50 \%$ time in each chamber) (Cunningham et al, 2006). The limitation of biased design is that an alternative interpretation of the data is that the drug decreases aversion to the non-preferred side rather than serving as a reinforcing stimulus in the $\mathrm{CPP}$ procedure (Bardo and Bevins, 2000; Mucha and Iversen, 1984; Mucha et al, 1982). Although the biased design may be susceptible to yielding false-positive results, it is also used due to absolute preference for the initially non-preferred side (Tzschentke, 1998). Moreover, cocaine and other psychoactive drugs produce CPP only when paired with the initially non-preferred side, but not with the initially preferred side (Nomikos and Spyraki, 1988; Tzschentke, 1998).

\section{FUNDING AND DISCLOSURE}

This work was supported by National Natural Sciences Foundation of China (81571301, 81271467, 81272459, 30970938). The authors declare no conflict of interest.

\section{ACKNOWLEDGMENTS}

We thank Miss Yuanyuan Xiao and Dr Chang Chen for assisting in the tandem mass spectrometry. We also thank $\mathrm{Dr}$ Chang Chen and Dr Jian-jun Chen for assisting in the statistical analysis. 


\section{AUTHOR CONTRIBUTIONS}

Conceived and designed the experiments: XC, LL and FFS. Performed the experiments: LL, HL and LJ. Analyzed the data: RZ, QZ, HG, JK, HL, WX, LJ. Contributed reagents/ materials/analysis tools: RZ, QZ, HG, JK, HL, WX, LJ, YZ. Wrote the manuscript: XC, LL and FFS. Revised: FFS and $\mathrm{XC}$. All authors read and approved the final manuscript.

\section{REFERENCES}

Aksoy S, Brandriff BF, Ward A, Little PF, Weinshilboum RM (1995). Human nicotinamide N-methyltransferase gene: molecular cloning, structural characterization and chromosomal localization. Genomics 29: 555-561.

Aksoy S, Szumlanski CL, Weinshilboum RM (1994). Human liver nicotinamide $\mathrm{N}$-methyltransferase. cDNA cloning, expression, and biochemical characterization. J Biol Chem 269: 14835-14840.

Anier K, Malinovskaja K, Aonurm-Helm A, Zharkovsky A, Kalda A (2010). DNA methylation regulates cocaine-induced behavioral sensitization in mice. Neuropsychopharmacology 35: 2450-2461.

Balleine BW, O'Doherty JP (2010). Human and rodent homologies in action control: corticostriatal determinants of goal-directed and habitual action. Neuropsychopharmacology 35: 48-69.

Bardo MT, Bevins RA (2000). Conditioned place preference: what does it add to our preclinical understanding of drug reward? Psychopharmacology (Berl) 153: 31-43.

Black YD, Maclaren FR, Naydenov AV, Carlezon WA Jr., Baxter MG, Konradi C (2006). Altered attention and prefrontal cortex gene expression in rats after binge-like exposure to cocaine during adolescence. J Neurosci 26: 9656-9665.

Bradke F, Dotti CG (2000). Establishment of neuronal polarity: lessons from cultured hippocampal neurons. Curr Opin Neurobiol 10: $574-581$.

Christian DT, Wang X, Chen EL, Sehgal LK, Ghassemlou MN, Miao JJ et al (2016). Dynamic alterations of rat nucleus accumbens dendritic spines over 2 months of abstinence from extendedaccess cocaine self-administration. Neuropsychopharmacology 42: 748-756.

Cunningham CL, Gremel CM, Groblewski PA (2006). Druginduced conditioned place preference and aversion in mice. Nat Protoc 1: 1662-1670.

Cushman I, Casey PJ (2009). Role of isoprenylcysteine carboxylmethyltransferase-catalyzed methylation in Rho function and migration. J Biol Chem 284: 27964-27973.

Cushman I, Casey PJ (2011). RHO methylation matters: a role for isoprenylcysteine carboxylmethyltransferase in cell migration and adhesion. Cell Adhesion Migration 5: 11-15.

Dietz DM, Sun H, Lobo MK, Cahill ME, Chadwick B, Gao V et al (2012). Rac1 is essential in cocaine-induced structural plasticity of nucleus accumbens neurons. Nat Neurosci 15: 891-896.

Everitt BJ, Robbins TW (2016). Drug addiction: updating actions to habits to compulsions ten years on. Annu Rev Psychol 67: 23-50.

Hancock JF, Hall A (1993). A novel role for RhoGDI as an inhibitor of GAP proteins. EMBO J 12: 1915-1921.

Hong S, Moreno-Navarrete JM, Wei X, Kikukawa Y, Tzameli I, Prasad D et al (2015). Nicotinamide N-methyltransferase regulates hepatic nutrient metabolism through Sirt1 protein stabilization. Nat Med 21: 887-894.

Jaffe AB, Hall A (2005). Rho GTPases: biochemistry and biology. Annu Rev Cell Dev Biol 21: 247-269.

Jayaram H, Hoelper D, Jain SU, Cantone N, Lundgren SM, Poy F et al (2016). S-adenosyl methionine is necessary for inhibition of the methyltransferase G9a by the lysine 9 to methionine mutation on histone H3. Proc Natl Acad Sci USA 113: 6182-6187.

Jones PA, Takai D (2001). The role of DNA methylation in mammalian epigenetics. Science 293: 1068-1070.
Jordi E, Heiman M, Marion-Poll L, Guermonprez P, Cheng SK, Nairn AC et al (2013). Differential effects of cocaine on histone posttranslational modifications in identified populations of striatal neurons. Proc Natl Acad Sci USA 110: 9511-9516.

Kim WY, Shin SR, Kim S, Jeon S, Kim JH (2009). Cocaine regulates ezrin-radixin-moesin proteins and RhoA signaling in the nucleus accumbens. Neuroscience 163: 501-505.

Kraus D, Yang Q, Kong D, Banks AS, Zhang L, Rodgers JT et al (2014). Nicotinamide N-methyltransferase knockdown protects against diet-induced obesity. Nature 508: 258-262.

Li J, Gu J, Wang B, Xie M, Huang L, Liu Y et al (2015a). Activation of dopamine D1 receptors regulates dendritic morphogenesis through Racl and RhoA in prefrontal cortex neurons. Mol Neurobiol 51: 1024-1037.

Li Y, Zhu R, Wang W, Fu D, Hou J, Ji S et al (2015b). Arginine methyltransferase 1 in the nucleus accumbens regulates behavioral effects of cocaine. J Neurosci 35: 12890-12902.

Li Z, Aizenman CD, Cline HT (2002). Regulation of rho GTPases by crosstalk and neuronal activity in vivo. Neuron 33: 741-750.

Liu W, Shang FF, Xu Y, Belegu V, Xia L, Zhao W et al (2015). eIF5A1/RhoGDIalpha pathway: a novel therapeutic target for treatment of spinal cord injury identified by a proteomics approach. Sci Rep 5: 16911.

Maze I, Nestler EJ (2011). The epigenetic landscape of addiction. Ann N Y Acad Sci 1216: 99-113.

Mucha RF, Iversen SD (1984). Reinforcing properties of morphine and naloxone revealed by conditioned place preferences: a procedural examination. Psychopharmacology (Berl) 82: 241-247.

Mucha RF, van der Kooy D, O'Shaughnessy M, Bucenieks P (1982). Drug reinforcement studied by the use of place conditioning in rat. Brain Res 243: 91-105.

Murray JE, Belin D, Everitt BJ (2012). Double dissociation of the dorsomedial and dorsolateral striatal control over the acquisition and performance of cocaine seeking. Neuropsychopharmacology 37: 2456-2466.

Nishimura A, Linder ME (2013). Identification of a novel prenyl and palmitoyl modification at the CaaX motif of Cdc42 that regulates RhoGDI binding. Mol Cell Biol 33: 1417-1429.

Nomikos GG, Spyraki C (1988). Cocaine-induced place conditioning: importance of route of administration and other procedural variables. Psychopharmacology (Berl) 94: 119-125.

Nyberg F (2014). Structural plasticity of the brain to psychostimulant use. Neuropharmacology 87: 115-124.

Parsons RB, Smith ML, Williams AC, Waring RH, Ramsden DB (2002). Expression of nicotinamide N-methyltransferase (E.C. 2.1.1.1) in the Parkinsonian brain. J Neuropathol Exp Neurol 61: 111-124.

Penzes P, Cahill ME, Jones KA, VanLeeuwen JE, Woolfrey KM (2011). Dendritic spine pathology in neuropsychiatric disorders. Nat Neurosci 14: 285-293.

Riederer M, Erwa W, Zimmermann R, Frank S, Zechner R (2009). Adipose tissue as a source of nicotinamide N-methyltransferase and homocysteine. Atherosclerosis 204: 412-417.

Roberts PJ, Mitin N, Keller PJ, Chenette EJ, Madigan JP, Currin RO et al (2008). Rho Family GTPase modification and dependence on CAAX motif-signaled posttranslational modification. J Biol Chem 283: 25150-25163.

Robinson TE, Kolb B (1999). Alterations in the morphology of dendrites and dendritic spines in the nucleus accumbens and prefrontal cortex following repeated treatment with amphetamine or cocaine. Eur J Neurosci 11: 1598-1604.

Saunderson EA, Spiers H, Mifsud KR, Gutierrez-Mecinas M, Trollope AF, Shaikh A et al (2016). Stress-induced gene expression and behavior are controlled by DNA methylation and methyl donor availability in the dentate gyrus. Proc Natl Acad Sci USA 113: 4830-4835. 
Shang F-F, Zhao W, Zhao Q, Liu J, Li D-W, Zhang H et al (2013). Upregulation of eIF-5A1 in the paralyzed muscle after spinal cord transection associates with spontaneous hindlimb locomotor recovery in rats by upregulation of the ErbB, MAPK and neurotrophin signal pathways. J Proteomics 91: 188-199.

Shang FF, Xia QJ, Liu W, Xia L, Qian BJ, You L et al (2016). miR-434-3p and DNA hypomethylation co-regulate eIF5A1 to increase AChRs and to improve plasticity in SCT rat skeletal muscle. Sci Rep 6: 22884.

Sperber H, Mathieu J, Wang Y, Ferreccio A, Hesson J, Xu Z et al (2015). The metabolome regulates the epigenetic landscape during naive-to-primed human embryonic stem cell transition. Nat Cell Biol 17: 1523-1535.

Thomas MG, Saldanha M, Mistry RJ, Dexter DT, Ramsden DB, Parsons RB (2013). Nicotinamide N-methyltransferase expression in SH-SY5Y neuroblastoma and N27 mesencephalic neurones induces changes in cell morphology via ephrin-B2 and Akt signalling. Cell Death Dis 4: e669.

Tiedje C, Sakwa I, Just U, Hofken T (2008). The Rho GDI Rdi1 regulates Rho GTPases by distinct mechanisms. Mol Biol Cell 19: 2885-2896.
Tzschentke TM (1998). Measuring reward with the conditioned place preference paradigm: a comprehensive review of drug effects, recent progress and new issues. Prog Neurobiol 56: 613-672.

Ulanovskaya OA, Zuhl AM, Cravatt BF (2013). NNMT promotes epigenetic remodeling in cancer by creating a metabolic methylation sink. Nat Chem Biol 9: 300-306.

Valjent E, Corbille AG, Bertran-Gonzalez J, Herve D, Girault JA (2006). Inhibition of ERK pathway or protein synthesis during reexposure to drugs of abuse erases previously learned place preference. Proc Natl Acad Sci USA 103: 2932-2937.

Wang GX, Zhang Y, Lv ZW, Sun M, Wu D, Chen XY et al (2014). Female specific association between NNMT gene and schizophrenia in a Han Chinese population. Int J Med Sci 11: 1234-1239.

Wang JQ (2003). Drugs of Abuse: Neurological Reviews and Protocols. Humana Press: Totowa, NJ, xiii: 514.

$\mathrm{Xu} \mathrm{Y,} \mathrm{Liu} \mathrm{P,} \mathrm{Zheng} \mathrm{DH,} \mathrm{Wu} \mathrm{N,} \mathrm{Zhu} \mathrm{L,} \mathrm{Xing} \mathrm{C} \mathrm{et} \mathrm{al} \mathrm{(2016).}$ Expression profile and prognostic value of NNMT in patients with pancreatic cancer. Oncotarget 7: 19975-19981.

Zhang J, Zheng YG (2016). SAM/SAH Analogs as Versatile Tools for SAM-Dependent Methyltransferases. ACS Chem Biol 11: 583-597.

Supplementary Information accompanies the paper on the Neuropsychopharmacology website (http://www.nature.com/npp) 